\title{
Ga-Tb (Gallium-Terbium)
}

\section{H. Okamoto}

The Ga-Tb phase diagram in [Masalski2] was redrawn from [Moffatt].

Figure 1 shows the Ga-Tb phase diagram calculated by [2008Jun]. The problem of asymmetry in the $\mathrm{Ga}_{2} \mathrm{~Tb}$ liquidus in the [Massalski2] diagram was solved.

[1981Cir] reported the existence of two modifications in the crystal structure of $\mathrm{Ga}_{3} \mathrm{~Tb}$ (Table 1). The transition temperature is unknown.

\section{References}

1981Cir: S. Cirafici and E. Franceschi, Stacking of Close-Packed $\mathrm{AB}_{3}$ Layers in $\mathrm{RGa}_{3}$ Compounds ( $\mathrm{R}=$ Heavy Rare Earth), $J$. Less-Common Met.,1981, 77, p 269-280

2008Jun: Z.K. Jun, L.B. Liu, H.S. Liu, X.M. Huang, and Z.P. Jin,

Thermodynamic Assessment of the Ga-Sc and Ga-Tb Systems, J. Alloys Compd., 2008, 463, p 511-515

Table 1 Ga-Tb crystal structure data

\begin{tabular}{|c|c|c|c|c|c|}
\hline Phase & Composition, at.\% Tb & Pearson symbol & Space group & Strukturbericht designation & Prototype \\
\hline$(\mathrm{Ga})$ & 0 & $o C 8$ & Cmca & $A 11$ & $\mathrm{Ga}$ \\
\hline $\mathrm{Ga}_{6} \mathrm{~Tb}$ & 14.3 & $t P 14$ & $P 4 / n b m$ & $\ldots$ & $\ldots$ \\
\hline$\beta \mathrm{Ga}_{3} \mathrm{~Tb}$ & 25 & $c P 4$ & $P m \overline{3} m$ & $L 1_{2}$ & $\mathrm{AuCu}_{3}$ \\
\hline$\alpha \mathrm{Ga}_{3} \mathrm{~Tb}$ & 25 & $h P 8$ & $\mathrm{~Pb}_{3} / m m c$ & $D 0_{19}$ & $\mathrm{Ni}_{3} \mathrm{Sn}$ \\
\hline $\mathrm{Ga}_{2} \mathrm{~Tb}$ & 33.3 & $h P 3$ & $P 6 / \mathrm{mmm}$ & $C 32$ & $\mathrm{AlB}_{2}$ \\
\hline $\mathrm{GaTb}$ & 50 & $o C 8$ & $\mathrm{Cmcm}$ & $B_{\mathrm{f}}$ & $\mathrm{CrB}$ \\
\hline $\mathrm{Ga}_{3} \mathrm{~Tb}_{5}$ & 62.5 & $t 132$ & $I 4 / \mathrm{mcm}$ & $D 8_{l}$ & $\mathrm{Cr}_{5} \mathrm{~B}_{3}$ \\
\hline$(\beta \mathrm{Tb})$ & 100 & $c I 2$ & $\operatorname{Im} \overline{3} m$ & $A 2$ & $\mathrm{~W}$ \\
\hline$(\alpha \mathrm{Tb})$ & 100 & $h P 4$ & $\mathrm{~Pb}_{3} / \mathrm{mmc}$ & $A 3$ & $\mathrm{Mg}$ \\
\hline
\end{tabular}

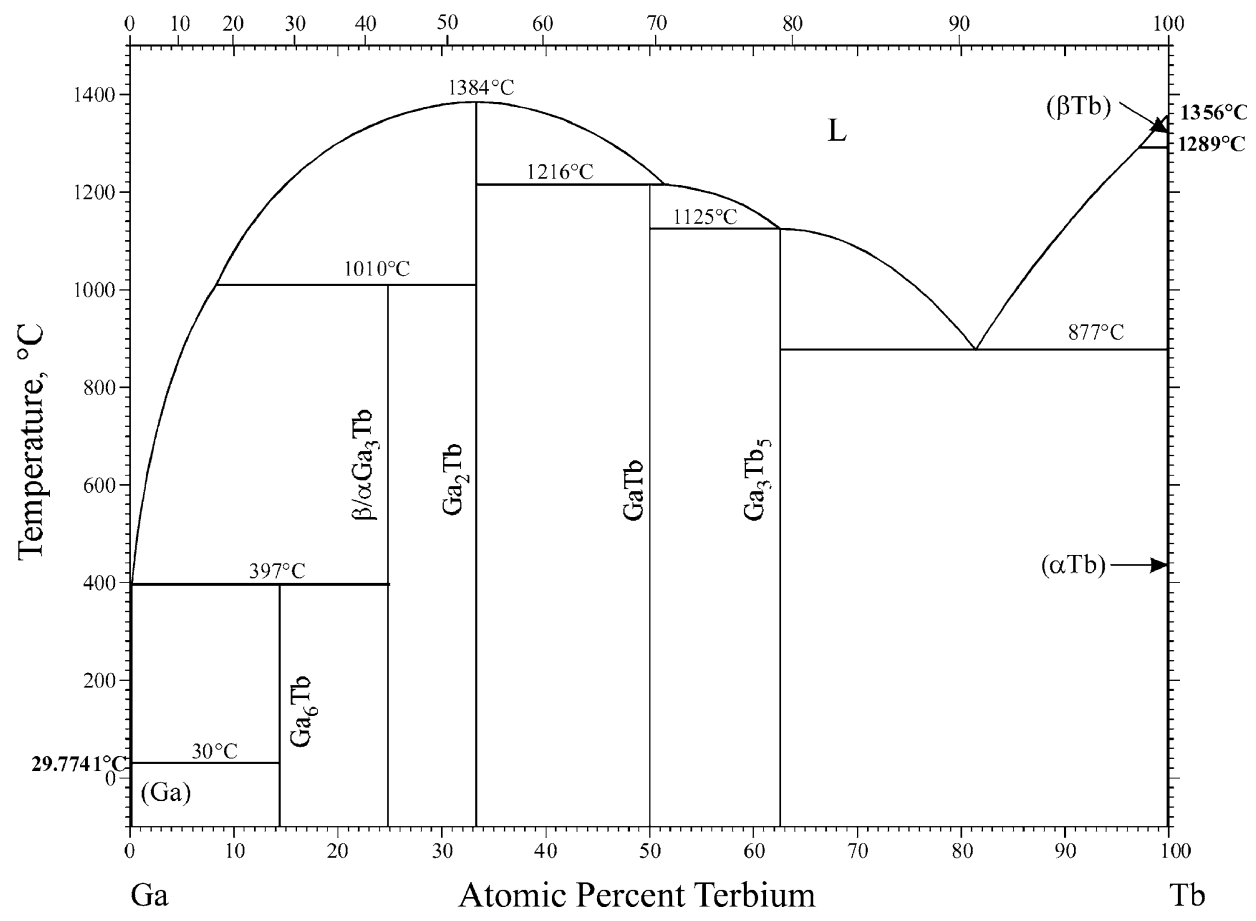

Fig. $1 \mathrm{Ga}-\mathrm{Tb}$ phase diagram 